\title{
Zoledronic acid significantly enhances radiation-induced apoptosis against human fibrosarcoma cells by inhibiting radioadaptive signaling
}

\author{
KAZUTAKA KOTO $^{1,2}$, HIROAKI MURATA ${ }^{1,3}$, SHINYA KIMURA $^{4}$, YASUSHI SAWAI ${ }^{1}$, \\ NAOYUKI HORIE ${ }^{1}$, TAKAAKI MATSUI ${ }^{1}$, KAZUTERU RYU ${ }^{1}$, EISHI ASHIHARA ${ }^{5}$, \\ TAIRA MAEKAWA ${ }^{5}$, TOSHIKAZU KUBO ${ }^{1}$ and SHINJI FUSHIKI ${ }^{2}$
}

\begin{abstract}
Departments of ${ }^{1}$ Orthopaedics and ${ }^{2}$ Pathology and Applied Neurobiology, Graduate School of Medical Science, Kyoto Prefectural University of Medicine, Kamigyo-ku, Kyoto 602-8566; ${ }^{3}$ Matsushita Memorial Hospital, Moriguchi, Osaka 570-8540; ${ }^{4}$ Division of Hematology, Respiratory Medicine and Oncology, Department of Internal Medicine, Faculty of Medicine, Saga University, Saga 849-8501; 5 Department of Transfusion Medicine and Cell Therapy, Kyoto University Hospital, Sakyo-ku, Kyoto 606-8507, Japan
\end{abstract}

Received September 13, 2012; Accepted October 30, 2012

DOI: $10.3892 /$ ijo.2012.1735

\begin{abstract}
Zoledronic acid (ZOL), a third-generation bisphosphonate, inhibits bone resorption, as well as exhibiting direct antitumor activity. To date, however, the combined effects of ZOL and ionizing radiation (IR) have not been assessed in patients with soft tissue sarcoma. We have, therefore, assessed the combined effects of ZOL and IR in fibrosarcoma cells. HT1080 fibrosarcoma cells were treated with ZOL and/or IR, together or sequentially and the antitumor effects were assessed. We found that ZOL significantly enhanced IR-induced apoptosis, especially when cells were treated with ZOL followed by IR. We, therefore, assessed the detailed mechanism of sequential treatment with ZOL and IR. Cells in $\mathrm{G} 2$ and $M$ phases, the most radiosensitive phases of the cell cycle, were not increased by low concentrations of ZOL. However, the levels of expression of Akt, ERK1/2 and NF- $\kappa \mathrm{B}$ proteins, all of which are related to radioadaptive resistance, were increased within a short time after irradiation with $3 \mathrm{~Gy}$, and this expression was inhibited by a low concentration of ZOL, which blocked the prenylation of small GTPases. This sequential treatment also increased the generation of reactive
\end{abstract}

Correspondence to: Dr Hiroaki Murata, Department of Orthopaedics, Graduate School of Medical Science, Kyoto Prefectural University of Medicine, 465 Kawaramachi-Hirokoji, Kamigyo-ku, Kyoto 602-8566, Japan

E-mail: murah@koto.kpu-m.ac.jp

Abbreviations: ZOL, zoledronic acid; IR, ionizing radiation; ROS, reactive oxygen species; BPs, bisphosphonates; DCFH-DA, 2,7-dichlorodihydrofluorescein diacetate; DMSO, dimethylsulfoxide

Key words: fibrosarcoma, zoledronic acid, radiation, combined effects, reactive oxygen species oxygen species (ROS). These results suggest that the combination of ZOL with IR may be beneficial in treating patients with soft tissue sarcoma.

\section{Introduction}

Fibrosarcoma is one of the high-grade malignant soft tissue sarcomas that commonly occurs in persons middle-aged and older. Fibrosarcoma involves deep soft tissues of the extremities and trunk. Although the prognosis of these patients has improved due to the development of surgical treatment and adjuvant chemotherapies, these therapies are not fully effective. The probability of local recurrence is related to the completeness of excision, with recurrence rates of $12-79 \%$ (1-3). Fibrosarcoma metastasizes to the lungs and bone. Metastasis occurs in 9-63\% of patients and the 5-year survival rate of these patients is $39-54 \%(2,3)$. To improve treatment outcomes, novel antitumor therapies are urgently required.

Bisphosphonates (BPs) are effective inhibitors of bone resorption and have been used in the treatment of metabolic bone diseases (4). Nitrogen-containing BPs (N-BPs), the so-called second- and third-generation BPs, induce apoptosis in osteoclasts by inhibiting protein prenylation in small $\mathrm{G}$ proteins through inhibition of farnesyl pyrophosphate synthase in the mevalonate pathway (5). It has been reported that thirdgeneration BPs, such as zoledronic acid (ZOL), the most potent N-BPs clinically available, may not only reduce bone loss, but may also exert direct antitumor effects against various malignant cells $(6,7)$. We also reported that the effects of ZOL against osteosarcoma cells and fibrosarcoma cells (8-11). However, ZOL is rapidly cleared from the circulation within 1-2 h (6). Furthermore, following infusion of the standard dose of ZOL, peak plasma levels were only $1-2 \mu \mathrm{M}$ (12). It is therefore likely that peripheral tumors are exposed to a low concentration of ZOL for only a few hours, and that the effects of ZOL alone may be insufficient in vivo. Therefore, combination therapy 
consisting of BPs along with other adjuvant therapy seemed to be required for treatment of soft tissue tumors.

There have been a number of reports regarding the combined effects of third-generation BPs with antitumor agents in various cancer cell lines $(10,13,14)$ and we have also reported that ZOL synergistically augments the effects of antitumor agents in fibrosarcoma cell lines (8). Another well-established treatment modality for the local treatment of malignant tumors is radiotherapy. There have been several recent reports regarding the combined effects of third-generation BPs with radiation in various cancer cell lines $(15,16)$. However, there have been no previous reports regarding the combined effects of ZOL with ionizing radiation (IR) in fibrosarcoma cells. Furthermore, there have been no reports concerning the detailed mechanisms underlying the combined effects even in other cancer cells. Therefore, this study was performed to clarify the combined effects of BPs and IR. It has been reported that cells in the G2 and M phases are more radiosensitive than those in other phases of the cell cycle $(17,18)$, so the effects of ZOL on the cell cycle were investigated in the present study. In addition, IR can also directly induce DNA damage causing double-strand breaks (DSB) and singlestrand breaks (19-21), and it also activates specific prosurvival signaling, including the MAPK pathway, PI3K-Akt pathway and $N F-\kappa B$ activation (21). It has also been reported that IR induces cell death by generating reactive oxygen species (ROS). Based on previous reports, we examined the inhibitory effects of ZOL on these signal pathways and the effects of cotreatment with ZOL and IR on ROS generation. Furthermore, taking clinical application into consideration, we evaluated the differences in antitumor effects according to dosage method and clarified one of the mechanisms of action in fibrosarcoma cells.

\section{Materials and methods}

Reagents. ZOL [1-hydroxy-2-(1H-imidazole-1-yl) ethylidenebisphosphonic acid] was obtained from Novartis Pharma AG (Basel, Switzerland). Akt inhibitor IV, Akt inhibitor VIII (Carbiochem, San Diego, CA), U0126 (Cell Signaling Technology, Beverly, MA), N-acetylcysteine (Nacalai Tesque Inc., Kyoto, Japan), the caspase inhibitors zVAD-fmk, zDEVD-fmk, zIETD-fmk, zLEHD-fmk and zAEVD-fmk (R\&D Systems, Minneapolis, MN) were purchased. Akt inhibitor IV, VIII, U0126, caspase inhibitors and N-acetylcysteine were dissolved in dimethyl sulfoxide (DMSO). An equivalent amount of DMSO was used as a control. The maximum volume (\%) of DMSO in the assays was $0.1 \%$.

$X$-ray irradiation. Cultured cells were irradiated with 0-8.0 Gy X-rays (Softex M-150WE; Softex Co. Ltd., Tokyo, Japan). The irradiation conditions selected were a distance of $1 \mathrm{~cm}$ from the focus to the specimen and an irradiation rate of $0.5 \mathrm{~Gy} / \mathrm{min}$ in air.

Cell lines and cell culture. The human fibrosarcoma cell line HT1080 was used. Cells were cultured in RPMI-1640 medium (Nacalai Tesque Inc.) supplemented with $10 \%$ fetal calf serum and $1 \%$ antibiotics.

Cell viability assay. Proliferation of the cell line was determined using the methylthiazol-diphenyl-tetrazolium (MTT) assay, as described previously (22). HT1080 cells were cultivated in flat- bottomed 96-well plates (Greiner Labortechnik, Frickenhausen, Germany) at $2 \times 10^{3}$ cells per well and incubated for $24 \mathrm{~h}$, followed by incubation with various concentrations/doses of ZOL and/or radiation for a further $72 \mathrm{~h}$. The mean of six data values for each treatment were calculated. The linear relationship between the degree of proliferation and cell number was evaluated within the range of the experiment. Half-maximal inhibitory concentrations $\left(\mathrm{IC}_{50}\right)$ were determined using the non-linear regression program CalcuSyn (Biosoft, Cambridge, UK).

Cell cycle analysis. To analyze alterations in the cell cycle, nuclear staining with propidium iodide (Sigma-Aldrich, Tokyo, Japan) was analyzed using a FACSCalibur flow cytometer (Becton-Dickinson, Franklin Lakes, NJ) as described previously (8). DNA histograms were created using Cell Quest software for Apple Macintosh (Becton-Dickinson). The ModFit LT V2.0 software (Verity Software, Topsham, ME) was used to analyze the data.

Detection of apoptosis. To analyze apoptosis, hypodiploid DNA (sub-G1) populations were assayed using a FACSCalibur flow cytometer as described previously (8).

Western blot analysis. Western blot analysis was performed as described previously (8) using antibodies to the following molecules: extracellular signal-regulated kinase (ERK1/2), phosphorylated ERK1/2 (p-ERK1/2), Akt, phosphorylated Akt (p-Akt), glyceraldehyde 3 phosphate dehydrogenase (GAPDH), caspase-3, -9, -10, cleaved caspase $-3,-9$ and phosphorylated Bad (p-Bad) (Cell Signaling Technology), Rap1A, cyclin B1 and cdc2 (Santa Cruz Biotechnology, Santa Cruz, CA), caspase-8 (Becton-Dickinson) and Bad (Assasy Designs Stressgen, Ann. Arbor, MI). The membranes were washed thoroughly and incubated for $1 \mathrm{~h}$ with horseradish peroxidase-conjugated anti-mouse or anti-rabbit IgG (Santa Cruz Biotechnology). Enhanced chemiluminescence (Amersham Biosciences, Tokyo, Japan) was used for detection. Optimized detection was achieved using the Chemi Doc ${ }^{\mathrm{TM}} \mathrm{XRS}^{+}$imaging system and Quantity One analysis software (Bio-Rad, Hercules, CA).

Transient transfection and luciferase assay. To determine promoter activity, we used a single-luciferase reporter assay system. HT1080 cells were plated in 24-well plates and incubated at $37^{\circ} \mathrm{C}$. At $70-80 \%$ confluence, the cells were washed and incubated with medium containing no serum or antibiotics for $6 \mathrm{~h}$. The cells were then transfected with the $\mathrm{NF}-\kappa \mathrm{B}$ reporter vector pGL4.32 (Promega, Madison, WI) using Lipofectamine 2000 (Invitrogen, Carlsbad, CA) reagent according to the manufacturer's protocol. Twenty-four hours after transfection, the cells were treated with medium containing $1.2 \mu \mathrm{M} \mathrm{ZOL}$, and $24 \mathrm{~h}$ after the start of treatment the cells were irradiated at $3 \mathrm{~Gy}$. One hour after irradiation, HT1080 cells were collected and lysed for luciferase assay using the ONE-Glo ${ }^{\mathrm{TM}}$ luciferase assay system (Promega). The light intensity was measured using a MicroLumat Plus LB96V (Berthold Technologies, Bad Wildbad, Germany). PSV- $\beta$ plasmid (Promega) was used as an internal control. All luciferase assays were carried out in triplicate.

Determination of intracellular ROS. Intracellular ROS levels were measured using 2,7-dichlorodihydrofluorescein diace- 
tate (DCFH-DA) (Nacalai Tesque Inc.) as a probe. Briefly, cells were loaded with DCFH-DA by incubation in complete medium containing $20 \mu \mathrm{M}$ DCFH-DA for $20 \mathrm{~min}$ in the dark at $37^{\circ} \mathrm{C}, 5 \% \mathrm{CO}_{2}$. The cells were rinsed with PBS, resuspended by trypsinization and analyzed by flow cytometry. The results were analyzed with Cell Quest software. Data are expressed as (number of positive cells) $\mathrm{x}$ (mean of the fluorescence intensity).

Statistical analysis. Data are expressed as means \pm SD of triplicate experiments. Statistical evaluation of the data was performed using Student's t-test for simple comparisons between groups and treatments. In all analyses, $\mathrm{P}<0.05$ was taken to indicate statistical significance.

\section{Results}

Co-treatment with ZOL and IR shows enhanced inhibitory effects on fibrosarcoma cell growth. The rate of growth inhibition was evaluated by MTT assay. The individual $\mathrm{IC}_{50}$ values for $\mathrm{ZOL}$ and radiation after $72 \mathrm{~h}$ of exposure were $1.77 \mu \mathrm{M}$ and $4.08 \mathrm{~Gy}$, respectively (Fig. 1A). Based on the $\mathrm{IC}_{50}$ values, we investigated the combined effects of $\mathrm{ZOL}$ at concentrations lower than the $\mathrm{IC}_{50}$ with radiation at a lower dose than the $\mathrm{IC}_{50}$.

Concurrent exposure (ZOL and IR): (CE). HT1080 cells were cultured and incubated for $24 \mathrm{~h}$, followed by incubation with various concentrations of $\mathrm{ZOL}$ combined with various doses of IR for a further $72 \mathrm{~h}$. The combined treatment induced significantly greater inhibitory effects than either used alone.

Sequential exposure (ZOL then IR): (SE). After $24 \mathrm{~h}$ of exposure to various concentrations of ZOL, cells were irradiated with various doses of X-rays. Each plate was evaluated a further $48 \mathrm{~h}$. The concentration/dose of ZOL/radiation were the same as in CE. Combined treatment induced significantly greater antitumor effects than either used alone (Fig. 1B).

Co-treatment with ZOL and IR enhances cytotoxic effects. The cytotoxic effects of co-treatment were evaluated using a FACSCalibur flow cytometer. Data from three independent experiments were collected. The combination method was as follows.

Concurrent exposure (ZOL and IR): (CE). HT1080 cells were cultured in 6 -well plates at $2 \times 10^{4}$ cells per well and incubated for $24 \mathrm{~h}$, followed by incubation with various concentrations of ZOL and/or IR at various doses. After a further $24 \mathrm{~h}$, cells were washed with PBS and incubated in fresh medium for a further $48 \mathrm{~h}$.

Sequential exposure (ZOL then IR): (SE I). After $24 \mathrm{~h}$ of incubation with various concentrations of ZOL, HT1080 cells were irradiated with X-rays, then washed with PBS and incubated in fresh medium for a further $48 \mathrm{~h}$.

Sequential exposure (IR then ZOL): (SE II). After $24 \mathrm{~h}$ of incubation with various doses of IR, HT1080 cells were incubated with various concentrations of ZOL for $24 \mathrm{~h}$. The cells were then washed with PBS and incubated in fresh medium for a further $24 \mathrm{~h}$. The sub-G1 fraction in flow cytometric analysis was increased by co-treatment with ZOL and IR, especially in SE I treatment (Fig. 1C).

Co-treatment with ZOL and IR induces apoptosis coupled with caspase activation and dephosphorylation of Bad. To confirm the cytotoxic effect of ZOL combined with IR, especially SE I, we carried out western blot analysis. After $24 \mathrm{~h}$ of incubation with $1.2 \mu \mathrm{M}$ ZOL, cells were irradiated at $3 \mathrm{~Gy}$, washed with PBS and incubated in fresh medium for a further $48 \mathrm{~h}$. Either ZOL or IR weakly affected Bad and caspases (Fig. 1D). However, ZOL and IR together resulted in marked cleavage of caspases-3, -9 and reduced caspases-8, -9. Moreover, co-treatment with ZOL and IR induced dephosphorylation of Bad.

Apoptosis induced by SE I treatment is blocked by caspase inhibitors. We also found that the pancaspase inhibitor zVAD-fmk and caspases-3, -8, -9 and -10 inhibitors, efficiently inhibited the sub-G1 population induced by SE I treatment. These results indicated that the apoptosis induced by co-treatment could be blocked by inhibition of caspases (Fig. 1E).

ZOL does not influence G2/M phase cells. To evaluate the antitumor mechanism of SE I treatment, cell cycle analysis was performed on HT1080 cells after $24 \mathrm{~h}$ of concurrent exposure to ZOL and IR. The results indicated an increase in number of cells in the $\mathrm{S}$ phase in a dose-dependent manner, but ZOL did not change the proportion of cells in the $\mathrm{G} 2 / \mathrm{M}$ phase. In addition, western blot analysis revealed that the levels of cyclin B1 and cdc2, which are G2/M phase-related proteins, were not altered after $24 \mathrm{~h}$ of exposure to ZOL. Furthermore, SE I treatment did not change the proportion of cells in $\mathrm{G} 2 / \mathrm{M}$ phase compared to each single treatment (Fig. 2).

ZOL inhibits prenylation of GTP-binding protein, and phosphorylation of Akt and ERK1/2 in HT1080 cells. To assess the involvement of the Akt pathway and MAPK pathway in apoptosis induced by the SE I treatment, the levels of several proteins were investigated by western blot analysis. The levels of unprenylated Rap1A, a small G protein located upstream of the PI3K-Akt pathway and MAPK pathway and a target of ZOL, were increased after $24 \mathrm{~h}$ of ZOL treatment in HT1080 cells (Fig. 3). P-ERK1/2 levels were decreased in a ZOL concentration-dependent manner and p-Akt levels began to decrease markedly $24 \mathrm{~h}$ after ZOL treatment.

Akt and ERK1/2 phosphorylated within a few hours after irradiation - ZOL pretreatment inhibits phosphorylation of these proteins. After $24 \mathrm{~h}$ of incubation with or without $1.2 \mu \mathrm{M}$ ZOL, HT1080 cells were washed with PBS, incubated in fresh medium and then irradiated at a dose of 3 Gy. After 10, $30 \mathrm{~min}, 1,3$, $6,12,24$ and $48 \mathrm{~h}$ of incubation, HT1080 cells were lysed and western blot analysis was performed. Irradiation at $3 \mathrm{~Gy}$ resulted in phosphorylation of Akt and ERK1/2 within $24 \mathrm{~h}$, especially at $1 \mathrm{~h}$, followed by normalization within $24 \mathrm{~h}$. This phosphorylation was significantly inhibited by pretreatment with $1.2 \mu \mathrm{M}$ ZOL compared to single radiation treatment alone (Fig. 4).

The cytotoxic effect was enhanced by co-treatment with IR and inhibition of Akt or MEK activity. Based on previous reports $(23,24)$ and the present findings, we hypothesized that the cytotoxic effects induced by SE I treatment may depend on the inhibition of Akt and/or ERK1/2 activity by ZOL. To investigate the inhibitory effects of ZOL on Akt and ERK1/2, HT1080 cells were exposed to Akt inhibitor IV, Akt inhibitor VIII, a selective inhibitor of Akt with no effect 
A)
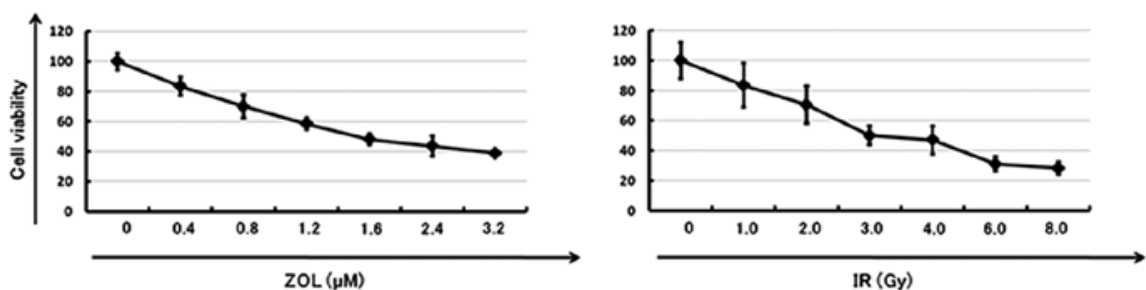

B)
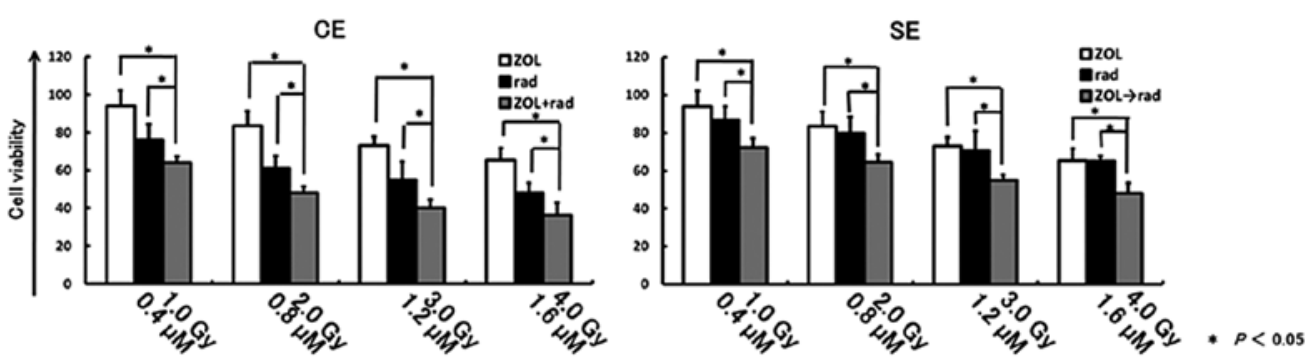

C)

CE

SE I

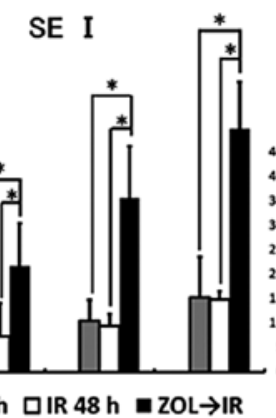

SE II

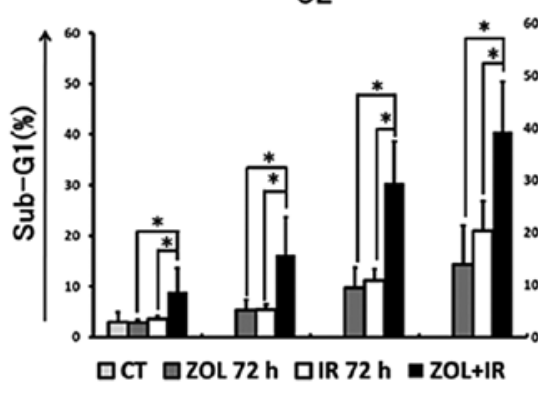

D)

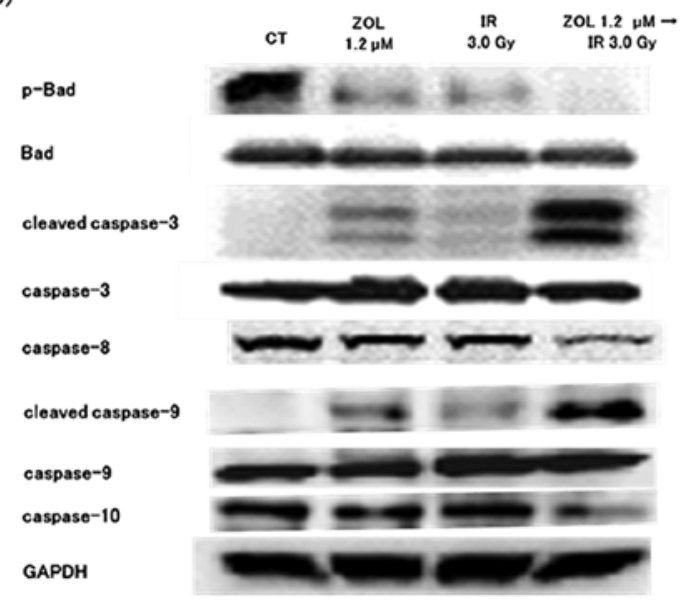

E)

Figure 1. SE I treatment-induced apoptosis is blocked by caspase inhibitors in HT1080 cells. (A) $\mathrm{IC}_{50}$ values of ZOL/IR were assessed by MTT assay. (B) Inhibitory effects of CE and SE treatment were assessed by MTT assay. (C) Cytotoxic effects of CE, SE I and SE II were evaluated by flow cytometry. (D) Protein level was evaluated by western blot analysis after ZOL/IR alone and SE I treatment. (E) Caspase inhibitors blocked the enhancement of IR-induced apoptosis by ZOL. HT1080 cells were treated with $1.2 \mu \mathrm{M}$ ZOL and/or 3 Gy IR for $24 \mathrm{~h}$ with or without various caspase inhibitors at $20 \mathrm{mM}$ : VAD, zVAD-fmk pancaspase inhibitor; C-3, zDEVD-fmk caspase-3 inhibitor; C-8, zIETD-fmk caspase-8 inhibitor; C-9, zLEHD-fmk caspase-9 inhibitor; C-10, zAEVD-fmk caspase-10 inhibitor. Apoptosis (sub-G1) was determined by flow cytometry.

on PI3K or PDK1, or U0126, a selective MEK inhibitor. After $24 \mathrm{~h}$ of incubation with these reagents, $\mathrm{p}$-Akt and p-ERK1/2 were detected by western blot analysis. We confirmed that p-Akt and p-ERK1/2 were markedly inhibited by $1.25 \mu \mathrm{M}$ Akt inhibitor IV (data are not shown), $1.5 \mu \mathrm{M}$ Akt inhibitor VIII (Fig. 5A), and $10.0 \mu \mathrm{M}$ U0126 (Fig. 5B). Next, we evaluated whether these inhibitors enhanced the cytotoxic effects of IR by flow cytometry. After $24 \mathrm{~h}$ of incubation with or without Akt inhibitor IV, VIII or U0126, cells were irradiated at a dose of $3 \mathrm{~Gy}$ and incubated for a further $48 \mathrm{~h}$. In cultures exposed to a combination of Akt inhibitor IV or VIII with IR, the sub-G1 population was significantly increased compared to either agent or IR alone (Fig. 5A) (data are not shown for Akt inhibitor IV). Sequential treatment with U0126 followed by IR tended to augment the sub-G1 population by IR, but the results were not statistically significant (Fig. 5B). 
A)

\section{ZOL treatment for $24 \mathrm{~h}$}

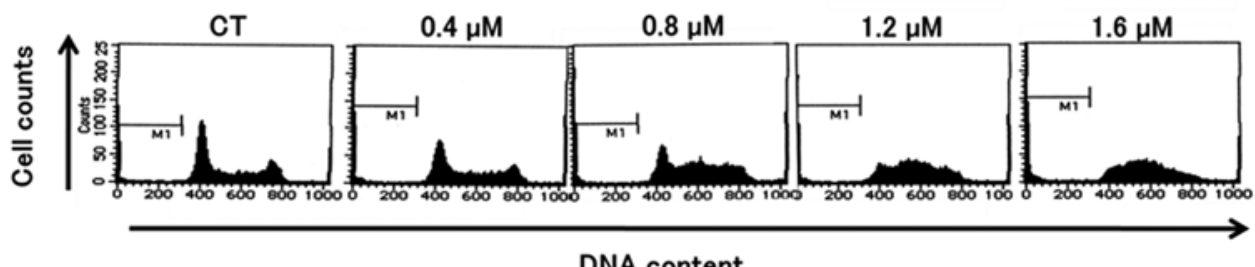

B)

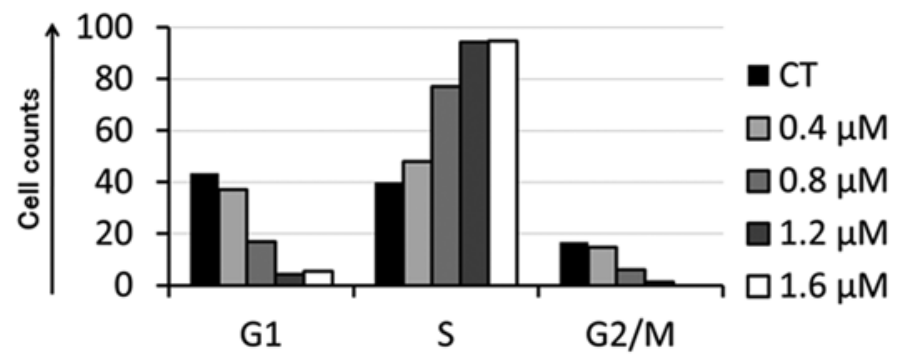

C)

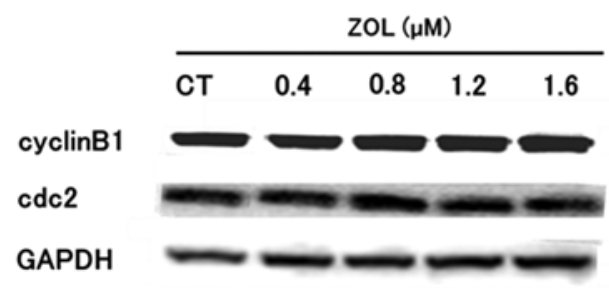

D)
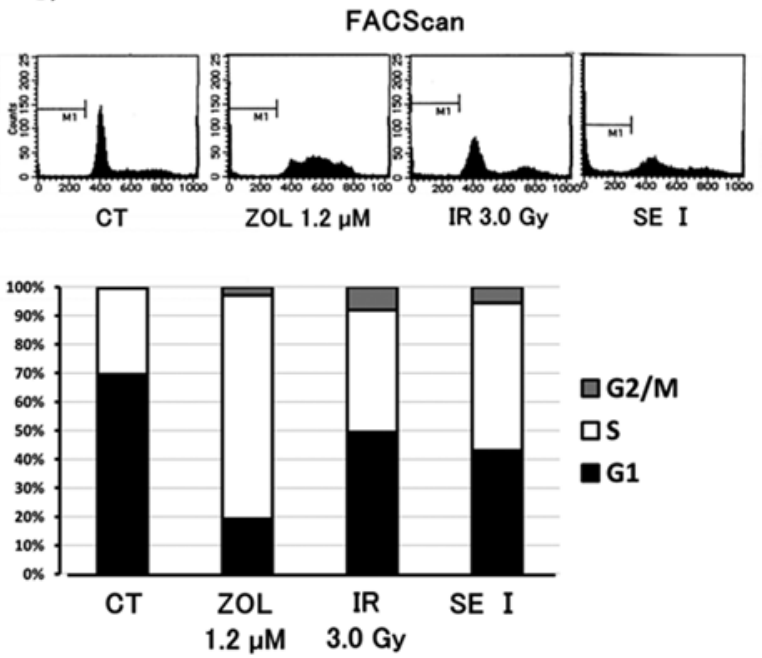

Figure 2. Effects of ZOL on cell cycle in HT1080 cells. Cells were treated with ZOL at the indicated concentrations for $24 \mathrm{~h}$. (A) The effect of ZOL on the cell cycle was evaluated by flow cytometry. (B) The DNA content was analyzed by flow cytometry. (C) Cell cycle-related proteins in HT1080 cells were evaluated by western blot analysis. Cells treated for $24 \mathrm{~h}$ with the indicated concentration of ZOL were lysed and analyzed. (D) SE I treatment did not influence the cell cycle compared to each single treatment.

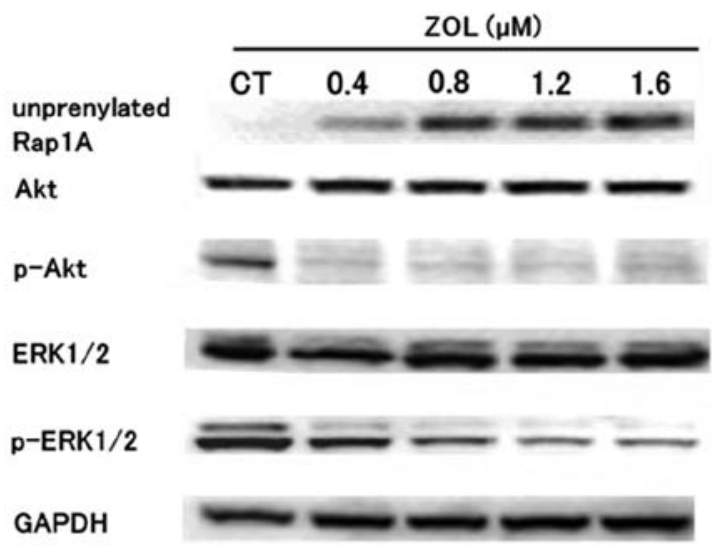

Figure 3. HT1080 cells treated with ZOL at the indicated concentrations for $24 \mathrm{~h}$ were lysed and analyzed by western blot analysis using antibodies that specifically recognize unprenylated Rap1A, Akt, p-Akt, ERK1/2 and p-ERK1/2.
Furthermore, the cell cycle was not altered by co-treatment with Akt inhibitor VIII or U0126 along with IR compared to each agent or IR alone.

$N F-\kappa B$ promoter activity stimulated by IR is inhibited by ZOL pretreatment. We investigated whether ZOL can inhibit the $\mathrm{NF}-\kappa \mathrm{B}$ gene promoter activity, which was reported to be activated by IR (25), using transient transfection with the NF- $\kappa$ B promoter-luciferase reporter plasmid, pGL4.32 or the empty vector PSV- $\beta$. Twenty-four hours after transfection, HT1080 cells were treated with or without medium containing ZOL and $24 \mathrm{~h}$ after the start of treatment, cells were irradiated at a dose of 0 or $3 \mathrm{~Gy}$. Then, $1 \mathrm{~h}$ after irradiation, cells were collected for luciferase assay. IR stimulated NF- $\kappa$ B promoter activity and ZOL inhibited the promoter activity compared to controls (Fig. 6). Furthermore, NF- $\kappa$ B promoter activity stimulated by IR was significantly inhibited by pretreatment with ZOL. 
A)

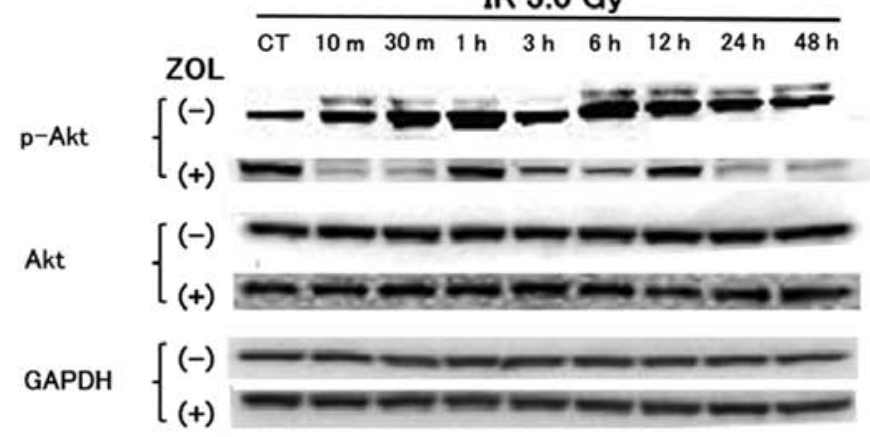

B)

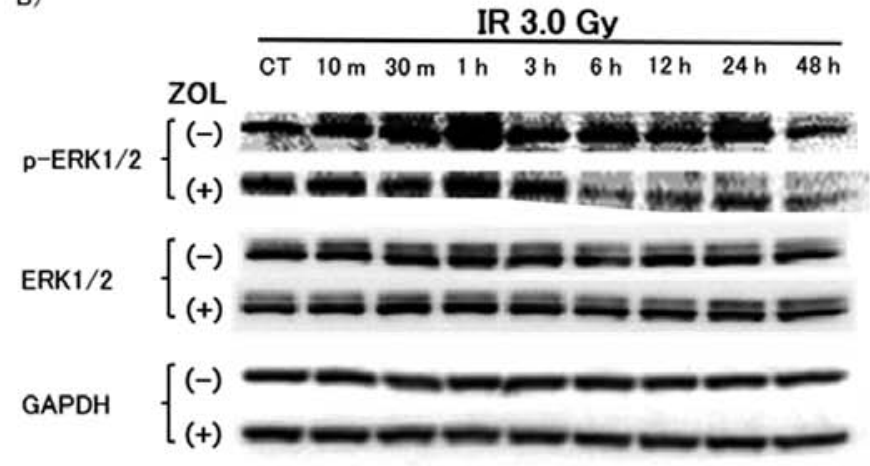

C)
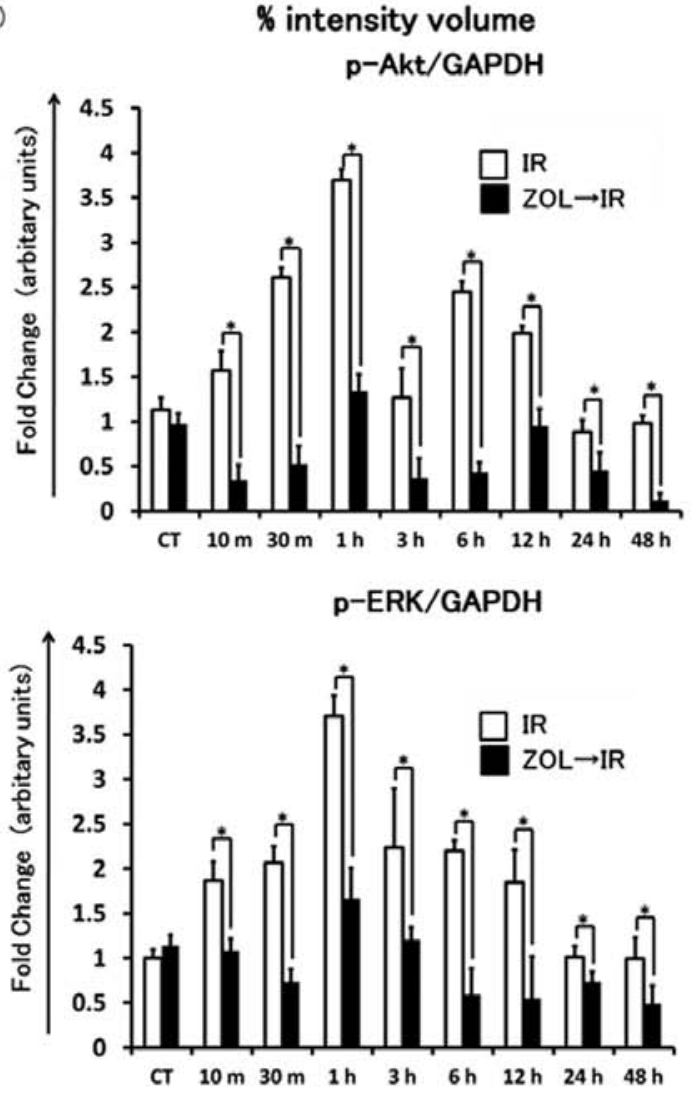

Figure 4. (A and B) Western blot analysis revealed phosphorylation of (A) Akt and (B) ERK1/2 by 3 Gy irradiation within 24 h and the phosphorylation was reduced by pretreatment with ZOL. CT indicates no treatment with ZOL and IR. (C) Percent intensity volume was calculated as mentioned above and normalized relative to GAPDH. Longitudinal axis, percentage intensity volume of each treatment/GAPDH.

ROS generation induced by IR is enhanced by ZOL, and the increasing cell death induced by SE I treatment is inhibited by $N$-acetylcysteine (NAC). To determine the involvement of ROS generation in cell death induced by SE I treatment in HT1080 cells, we monitored ROS generation by flow cytometry. After $24 \mathrm{~h}$ of incubation, cells $\left(2 \times 10^{4}\right)$ were treated with various concentrations of ZOL in the presence or absence of $1 \mathrm{mMNAC}$ for $24 \mathrm{~h}$, then exposed to IR. Forty-eight hours after IR, cells were incubated with DCFH-DA, and the fluorescence intensity was measured by flow cytometry. In comparison to IR alone or ZOL alone, ROS generation was significantly increased after SE I treatment and the ROS generation was inhibited by NAC treatment. Furthermore, the sub-G1 population in the HT1080 cells treated with NAC was significantly reduced compared to the untreated control group (Fig. 7).

\section{Discussion}

New treatment strategies are necessary to improve the prognosis of soft tissue sarcoma. In the search for new treatment strategies and antitumor agents, as well as from our previous studies $(8-11,26)$, we found that ZOL was a potent enhancer of radiation-induced apoptosis in fibrosarcoma cells. First, we compared three patterns of administration, i.e., CE, SE I, and SE II, to determine the most effective dosage method for clinical application. The results indicated that SE I treatment exerted the most potent antitumor effects, so we performed further investigation of the cytotoxic effects of SE I. The increase in proportion of cells in sub-G1, and the induction of caspase-3 cleavage by SE I treatment suggested that it induced apoptosis, and we assumed that apoptosis was induced through not only the mitochondrial apoptotic pathway but also via the death receptor pathway because caspase- $8,-9$ and -10 inhibitors decreased the proportion of cells in sub-G1.

Next, we examined the mechanisms underlying the cytotoxic effects of SE I treatment. Based on previous reports that cells in the $\mathrm{G} 2$ and $\mathrm{M}$ phases are more radiosensitive than those in other phases of the cell cycle $(17,18)$, we hypothesized that synergistic effects could be obtained if ZOL arrested the cell cycle in $\mathrm{G} 2 / \mathrm{M}$ phases. However, the proportion of cells in $\mathrm{G} 2 / \mathrm{M}$ phase was not increased by ZOL.

Based on our results, we investigated another mechanism for the cytotoxic effects of SE I treatment. Whereas IR is an effective treatment for malignant tumor cells by directly causing DNA damage $(27,28)$, radioadaptive resistance, a specific prosurvival signaling network or radioprotective mechanisms activated by IR, has also been reported $(21,29)$. The term radioadaptive response was originally used to describe a reduced cell sensitivity to a higher challenge dose when a smaller inducing radiation dose had been applied earlier (30). NF- $\kappa \mathrm{B}$ is a transcription factor that plays a key role in tumor radioadaptive resistance. It has been reported that DNA binding of NF- $\kappa \mathrm{B}$ was 
A)

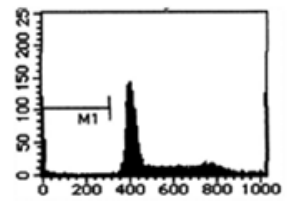

CT

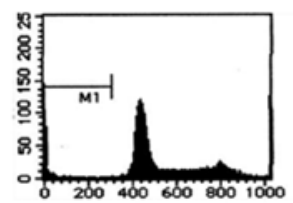

Akt inhibitor VIII $1.5 \mu \mathrm{M}$

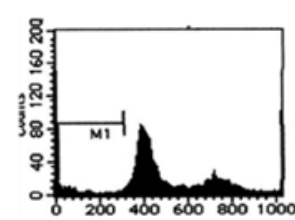

IR $3.0 \mathrm{~Gy}$
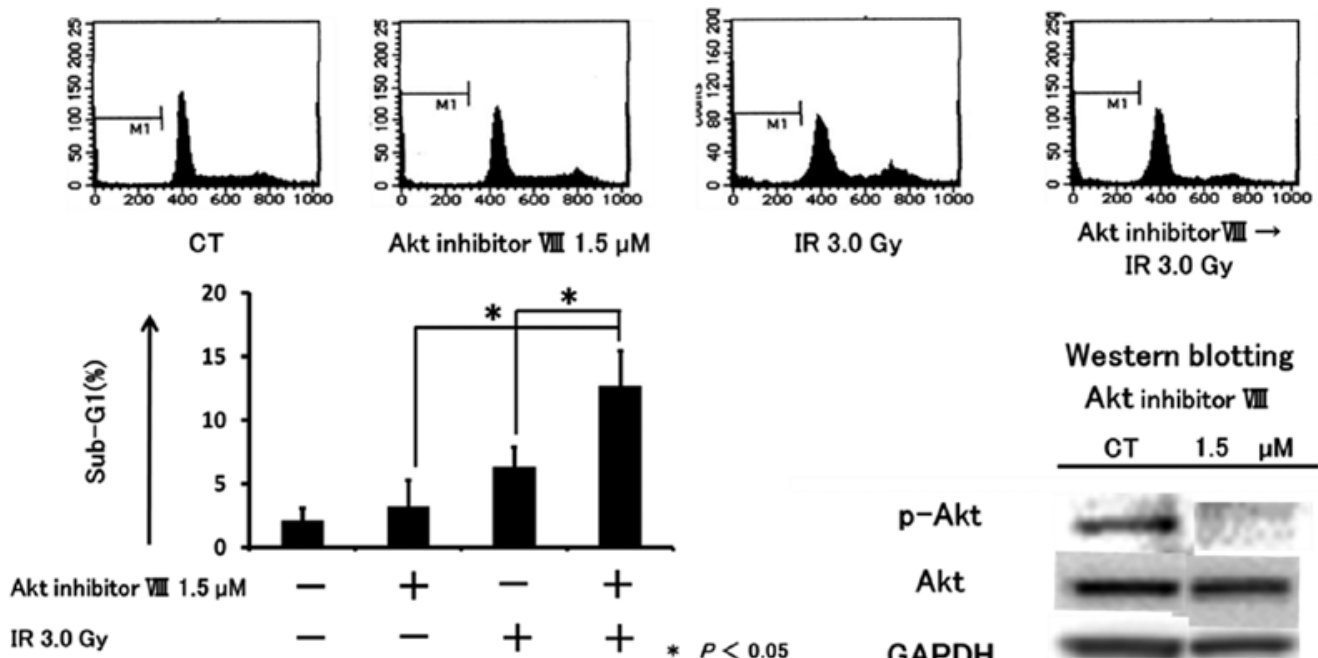

Akt inhibitorVII $\rightarrow$

IR 3.0 Gy

\section{Western blotting}

Akt inhibitor VII

CT $\quad 1.5 \quad \mu \mathrm{M}$

p-Akt

Akt

GAPDH

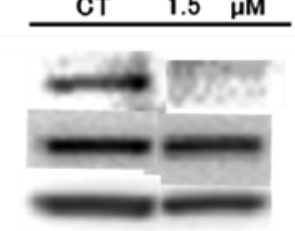

B)

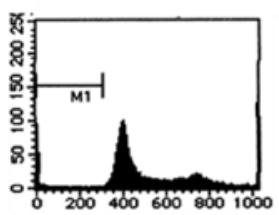

CT

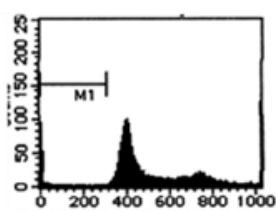

U0126 $10.0 \mu \mathrm{M}$

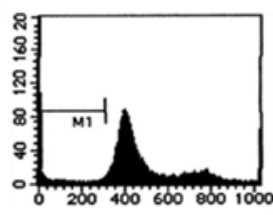

IR 3.0 Gy

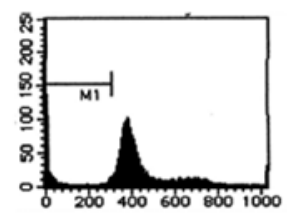

$\mathrm{U} 0126 \rightarrow$ IR 3.0 Gy

Western blotting

U0126

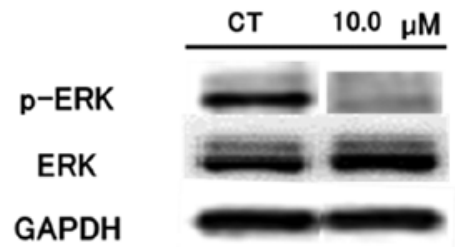

Figure 5. Akt inhibitor VIII and MEK inhibitor U0126 augment the cell death caused by IR. (A) Akt inhibitor VIII. (B) U0126. HT1080 cells were exposed to DMSO, Akt inhibitor VIII $(1.5 \mu \mathrm{mol} / \mathrm{l})$, U0126 $(10.0 \mu \mathrm{M})$, IR (3 Gy) or sequential treatment with Akt inhibitor VIII or U0126 followed by IR. Western blot analysis showed that each agent inhibited the phosphorylation of Akt or ERK1/2. Equivalent DMSO was used as a control (CT). Data are shown as means (bars, SD) (n=3). ${ }^{*}<0.05$.

A)

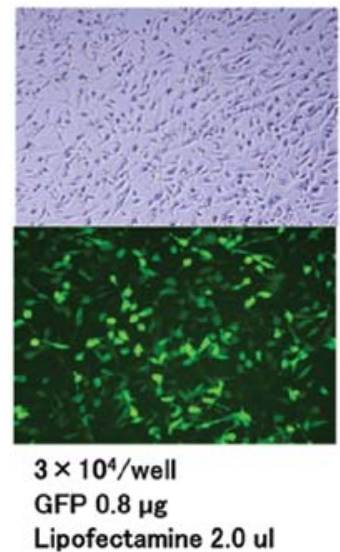

B)

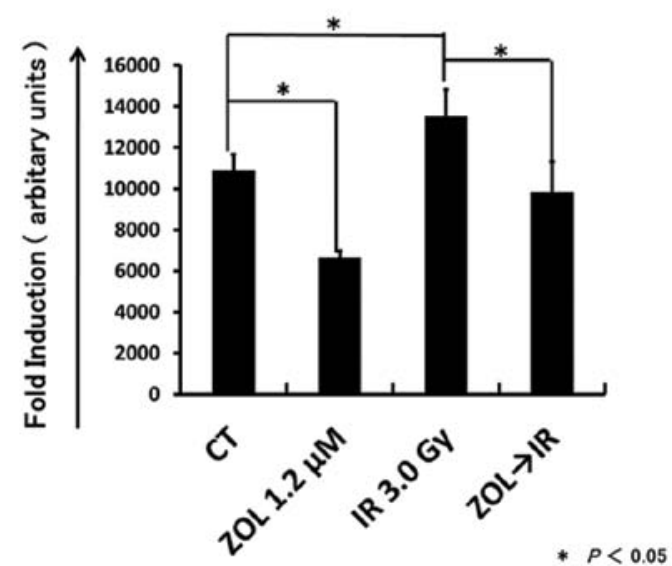

Figure 6. NF-kB promoter activity stimulated by IR was inhibited by pretreatment with ZOL in HT1080 cells. (A) Green fluorescent protein (GFP) was transfected to confirm the efficacy of plasmid transfection. (B) HT1080 cells were transiently transfected with the NF- $\mathrm{KB}$ reporter plasmid, and luciferase activity was measured after incubation with ZOL alone, IR alone or SE I treatment. The raw light units (RLU) from the cell lysates were standardized relative to the protein concentrations. The fold induction by each treatment was calculated relative to that of DMSO and indicated on the right. Data are shown as means $($ bars, SD) $(n=3)$. " $P<0.05$. 
A)

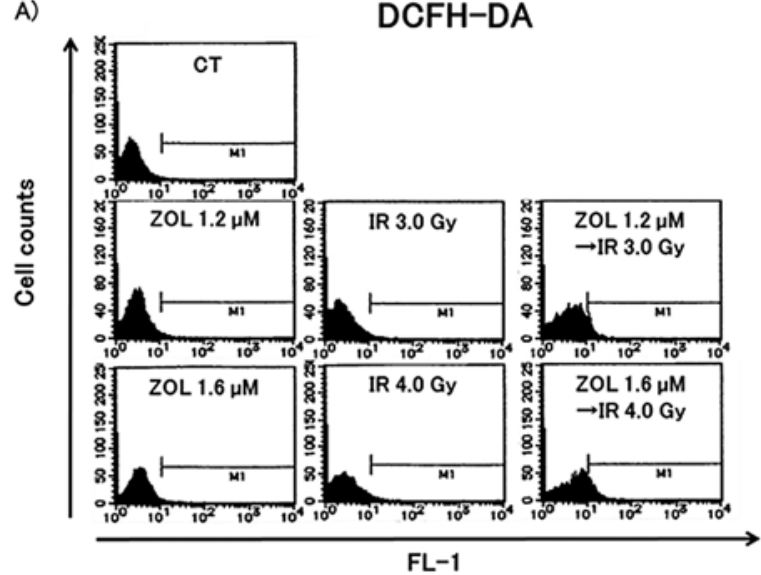

B)

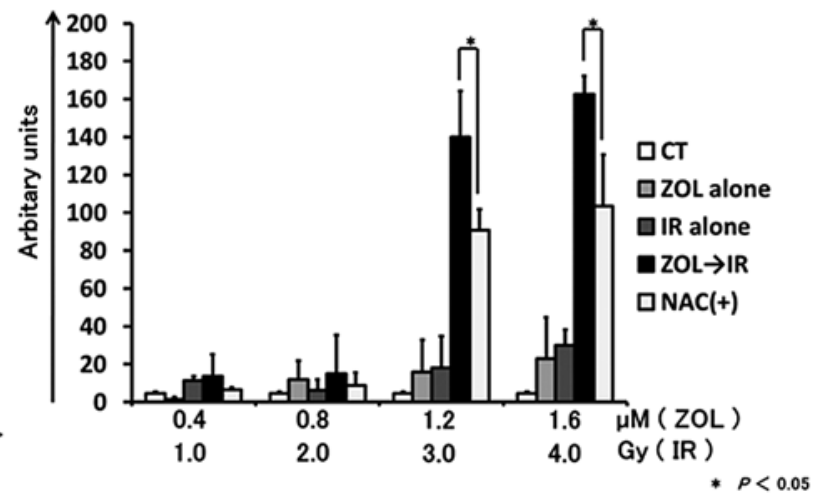

c)

N-Acetylecystein ( NAC )

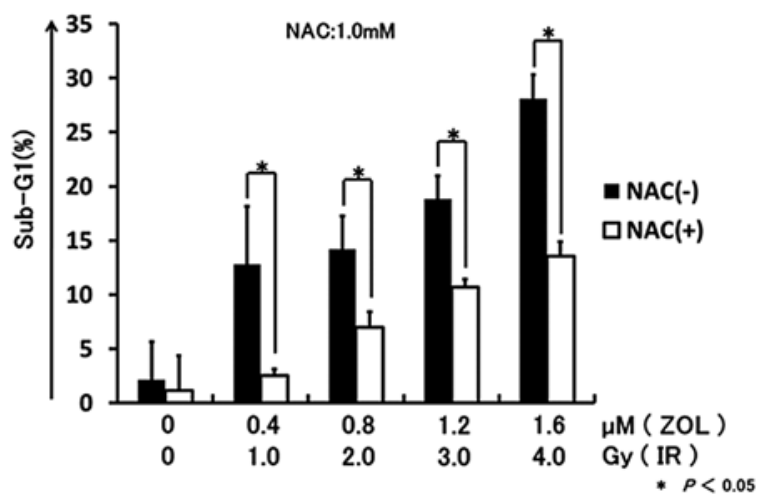

Figure 7. (A) ROS generation is increased by SE I treatment. (B) NAC inhibited ROS generation caused by SE I treatment. Data are expressed as (number of FL1positive cells) $x$ (mean fluorescence intensity). (C) The increase in sub-G1 fraction induced by SE I treatment was reduced by NAC in HT1080 cells. Equivalent DMSO was used as a control (CT). Data are shown as means (bars, SD) $(n=3)$. IR, ionizing radiation.

activated by IR $(31,32)$ and was regulated by various signaling cascades, e.g., the TNF- $\alpha$ pathway, Ras-PI3K-Akt pathway, and Ras-MAPK pathway (33-36). In addition, several genes, such as the anti-apoptotic Bcl family, cyclin B1, cyclin D2, superoxide dismutases (SOD) that suppress ROS generation and HER-2, were identified as effector genes of NF- $\mathrm{KB}$ and these upregulated both cell proliferation and viability (21). Furthermore, it has been reported that blocking NF- $\mathrm{KB}$ activation increases the apoptotic response and decreases growth and clonogenic survival of several human cancer cell lines $(37,38)$. In the present study, we evaluated the inhibitory effects of ZOL on radioadaptive signaling, which was unfavorable in treatment of malignant tumor cells. We have reported previously that ZOL inhibited proliferation and induced apoptosis in HT1080 cells by inhibiting activation of small $\mathrm{G}$ protein prenylation (8). In the present study, we showed that ZOL alone inhibited phosphorylation of Akt and ERK1/2, which are downstream of the PI3K-Akt pathway and MAPK pathway. Furthermore, pretreatment with ZOL also inhibited phosphorylation of Akt and ERK1/2, which were activated by IR and combined treatment with IR and MEK inhibitor/Akt inhibitors augmented the cytotoxic effects in HT1080 cells. These results supported the findings of recent studies that inhibition of the Ras-PI3K-Akt pathway or Ras-MAPK pathway enhanced the cytotoxic effects of IR (39-41). These results suggested that ZOL inhibited PI3K-Akt signaling or MAPK signaling via the inhibition of small $\mathrm{G}$ protein prenylation as one mechanism underlying the combined effects of ZOL and IR treatment. In addition, ZOL markedly inhibited Akt phosphorylation. These results suggested that ZOL may directly inhibit phosphorylation of Akt by regulation of the inositol phospholipid pathway, which is upstream of AKT, and not via its effects on small GTPases, because ZOL negatively regulates lipid metabolism.

In the present study, IR induced Akt and ERK1/2 phosphorylation in HT1080 cells within $24 \mathrm{~h}$ (especially at $1 \mathrm{~h}$ ) after irradiation, similar to the findings of previous studies $(23,24)$. Furthermore, we showed that pretreatment with ZOL also inhibited the activation of NF-kB in HT1080 cells, although transcription of NF- $\kappa \mathrm{B}$ was activated $1 \mathrm{~h}$ after irradiation. These results suggested a mechanism by which SE I was the most effective treatment as follows. SE I treatment induced apoptosis synergistically by ZOL inhibition of the phosphorylation of Akt, ERK1/2 and activation of NF- $\mathrm{kB}$, which are involved in radioadaptive signaling, and were upregulated within a very short time. However, it has been reported that treatment of cells with a MEK/ERK inhibitor shows either little or no effect on 
IR-induced apoptosis $(42,43)$. Therefore, the role of ERK activation on cell radiosensitivity has not been clarified, and further investigations are necessary.

On the other hand, the term ROS refers to a group of molecules such as peroxides and free radicals derived from oxygen that are highly reactive toward biomolecules. ROS are produced not only by endogenous sources, but also by exogenous sources, such as IR, chemicals, toxins and pollutants (44). Elevated ROS levels can create oxidative stress in the cell and chronic exposure to this stress can result in permanent changes in the genome (45). It is well known that the large and sudden increase in ROS generation in cells by IR can lead to apoptotic cell death (46). Excessive ROS, e.g., that generated by IR, induces mitochondrial apoptosis, because mitochondrial DNA is highly sensitive to mutations caused by endogenous ROS $(47,48)$. DSB by ROS can arise when ROS-induced DNA damage interferes with either DNA replication or transcription $(49,50)$. In the present study, intracellular ROS levels in HT1080 cells were increased by SE I treatment, and the cytotoxic effects enhanced by co-treatment with ZOL were reduced by NAC treatment. These results suggested that ROS generation played a significant role in the combined effects of ZOL with IR. The mechanism was thought to be as follows. As a result of unprenylation of small GTPase by ZOL, NF- $\kappa \mathrm{B}$ was inactivated via inhibition of the PI3K-Akt pathway and MAPK pathway downstream of small GTPases and then ROS generation was increased by inhibition of NF- $\mathrm{BB}$ effector genes, e.g., MnSOD and CuSOD. However, further studies are required as it is difficult to clarify the precise mechanism underlying the combined effect based only on our results.

We showed for the first time that ZOL significantly enhances radiation-induced apoptosis in human fibrosarcoma cells. Although the detailed mechanism has not been reported, we demonstrated one of the mechanisms underlying the synergistic effects of ZOL and IR. Due to inhibition of small GTPase prenylation, ZOL inhibited phosphorylation of AKT and ERK1/2, along with the transcriptional activity of NF- $\kappa$ B, and synergistic effects were obtained. Furthermore, SE I was postulated to be the most effective treatment because adaptive resistance to IR occurred within a few hours. The increased ROS generation was also one of the mechanisms underlying the combined effects. Although further studies of the in vivo effects are required, these results raise the possibility that the combination of ZOL and radiation may represent a promising type of therapy for fibrosarcoma.

\section{Acknowledgements}

This study was supported by JSPS KAKENHI (Grant-in-Aid for Scientific Research C: 23592196 to HM).

\section{References}

1. Pritchard DJ, Sim FH, Ivins JC, Soule EH and Dahlin DC: Fibrosarcoma of bone and soft tissues of the trunk and extremities. Orthop Clin North Am 8: 869-881, 1977.

2. Pritchard DJ, Soule EH, Taylor WF and Ivins JC: Fibrosarcoma - a clinicopathologic and statistical study of 199 tumors of the soft tissues of the extremities and trunk. Cancer 33: 888-897, 1974.

3. Scott SM, Reiman HM, Pritchard DJ and Ilstrup DM: Soft tissue fibrosarcoma. A clinicopathologic study of 132 cases. Cancer 64: 925-931, 1989.
4. Russell RG and Rogers MJ: Bisphosphonates: from the laboratory to the clinic and back again. Bone 25: 97-106, 1999.

5. Green JR: Antitumor effects of bisphosphonates. Cancer 97: 840-847, 2003

6. Lee MV, Fong EM, Singer FR and Guenette RS: Bisphosphonate treatment inhibits the growth of prostate cancer cells. Cancer Res 61: 2602-2608, 2001.

7. Kubo T, Shimose S, Matsuo T, et al: Inhibitory effects of a new bisphosphonate, minodronate, on proliferation and invasion of a variety of malignant bone tumor cells. J Orthop Res 24: 1138-1144, 2006.

8. Koto K, Murata H, Kimura S, et al: Zoledronic acid inhibits proliferation of human fibrosarcoma cells with induction of apoptosis, and shows combined effects with other anticancer agents. Oncol Rep 24: 233-239, 2010.

9. Horie N, Murata H, Nishigaki Y, et al: The third-generation bisphosphonates inhibit proliferation of murine osteosarcoma cells with induction of apoptosis. Cancer Lett 238: 111-118, 2006

10. Horie N, Murata H, Kimura S, et al: Combined effects of a third-generation bisphosphonate, zoledronic acid with other anticancer agents against murine osteosarcoma. $\mathrm{Br} \mathrm{J}$ Cancer 96: 255-261, 2007.

11. Koto K, Horie N, Kimura S, et al: Clinically relevant dose of zoledronic acid inhibits spontaneous lung metastasis in a murine osteosarcoma model. Cancer Lett 274: 271-278, 2009.

12. Jagdev SP, Coleman RE, Shipman CM, Rostami HA and Croucher PI: The bisphosphonate, zoledronic acid, induces apoptosis of breast cancer cells: evidence for synergy with paclitaxel. Br J Cancer 84: 1126-1134, 2001.

13. Kubo T, Shimose S, Matsuo T, Sakai A and Ochi M: Efficacy of a nitrogen-containing bisphosphonate, minodronate, in conjunction with a p38 mitogen activated protein kinase inhibitor or doxorubicin against malignant bone tumor cells Cancer Chemother Pharmacol 62: 111-116, 2008.

14. Ottewell PD, Monkkonen H, Jones M, Lefley DV, Coleman RE and Holen I: Antitumor effects of doxorubicin followed by zoledronic acid in a mouse model of breast cancer. J Natl Cancer Inst 100: 1167-1178, 2008.

15. Ural AU, Avcu F, Candir M, Guden M and Ozcan MA: In vitro synergistic cytoreductive effects of zoledronic acid and radiation on breast cancer cells. Breast Cancer Res 8: R52, 2006.

16. Algur E, Macklis RM and Hafeli UO: Synergistic cytotoxic effects of zoledronic acid and radiation in human prostate cancer and myeloma cell lines. Int J Radiat Oncol Biol Phys 61: 535-542, 2005.

17. Milas L, Hunter NR, Mason KA, Kurdoglu B and Peters LJ: Enhancement of tumor radioresponse of a murine mammary carcinoma by paclitaxel. Cancer Res 54: 3506-3510, 1994.

18. Tishler RB, Geard CR, Hall EJ and Schiff PB: Taxol sensitizes human astrocytoma cells to radiation. Cancer Res 52: 3495-3497, 1992.

19. Acharya A, Das I, Chandhok D and Saha T: Redox regulation in cancer: a double-edged sword with therapeutic potential. Oxid Med Cell Longev 3: 23-34, 2010.

20. Sedelnikova OA, Redon CE, Dickey JS, Nakamura AJ, Georgakilas AG and Bonner WM: Role of oxidatively induced DNA lesions in human pathogenesis. Mutat Res 704: 152-159, 2010.

21. Ahmed KM and Li JJ: NF-kappa B-mediated adaptive resistance to ionizing radiation. Free Radic Biol Med 44: 1-13, 2008

22. Hansen MB, Nielsen SE and Berg K: Re-examination and further development of a precise and rapid dye method for measuring cell growth/cell kill. J Immunol Methods 119: 203-210, 1989

23. Ram R, Uziel O, Eldan O, et al: Ionizing radiation up-regulates telomerase activity in cancer cell lines by post-translational mechanism via ras/phosphatidylinositol 3-kinase/Akt pathway. Clin Cancer Res 15: 914-923, 2009.

24. Zingg D, Riesterer O, Fabbro D, Glanzmann C, Bodis S and Pruschy M: Differential activation of the phosphatidylinositol 3'-kinase/Akt survival pathway by ionizing radiation in tumor and primary endothelial cells. Cancer Res 64: 5398-5406, 2004.

25. Wang CY, Mayo MW and Baldwin AS Jr: TNF- and cancer therapy-induced apoptosis: potentiation by inhibition of NF-kappaB. Science 274: 784-787, 1996.

26. Ryu K, Murata H, Koto K, et al: Combined effects of bisphosphonate and radiation on osteosarcoma cells. Anticancer Res 30: 2713-2720, 2010. 
27. Morgan WF and Murnane JP: A role for genomic instability in cellular radioresistance? Cancer Metastasis Rev 14: 49-58, 1995.

28. Morgan WF: Is there a common mechanism underlying genomic instability, bystander effects and other nontargeted effects of exposure to ionizing radiation? Oncogene 22: 70947099, 2003.

29. Ch'ang HJ, Maj JG, Paris F, et al: ATM regulates target switching to escalating doses of radiation in the intestines. Nat Med 11: 484-490, 2005.

30. Stecca $\mathrm{C}$ and Gerber GB: Adaptive response to DNA-damaging agents: a review of potential mechanisms. Biochem Pharmacol 55: 941-951, 1998.

31. Spitz DR, Azzam EI, Li JJ and Gius D: Metabolic oxidation/reduction reactions and cellular responses to ionizing radiation: a unifying concept in stress response biology. Cancer Metastasis Rev 23: 311-322, 2004.

32. Schieven GL, Kirihara JM, Myers DE, Ledbetter JA and Uckun FM: Reactive oxygen intermediates activate NF-kappa $\mathrm{B}$ in a tyrosine kinase-dependent mechanism and in combination with vanadate activate the p56lck and p59fyn tyrosine kinases in human lymphocytes. Blood 82: 1212-1220, 1993.

33. Guo G, Wang T, Gao Q, et al: Expression of ErbB2 enhances radiation-induced NF-kappaB activation. Oncogene 23: $535-545,2004$

34. Blonska M, You Y, Geleziunas R and Lin X: Restoration of NF-kappaB activation by tumor necrosis factor alpha receptor complex-targeted MEKK3 in receptor-interacting proteindeficient cells. Mol Cell Biol 24: 10757-10765, 2004.

35. Chen G and Goeddel DV: TNF-R1 signaling: a beautiful pathway. Science 296: 1634-1635, 2002.

36. Dent P, Yacoub A, Fisher PB, Hagan MP and Grant S: MAPK pathways in radiation responses. Oncogene 22: 5885-5896, 2003.

37. Tang G, Minemoto Y, Dibling B, et al: Inhibition of JNK activation through NF-kappaB target genes. Nature 414: 313-317, 2001.

38. Chen X, Shen B, Xia L, et al: Activation of nuclear factor kappaB in radioresistance of TP53-inactive human keratinocytes. Cancer Res 62: 1213-1221, 2002.

39. Kim IA, Bae SS, Fernandes A, et al: Selective inhibition of Ras, phosphoinositide 3 kinase, and Akt isoforms increases the radiosensitivity of human carcinoma cell lines. Cancer Res 65 7902-7910, 2005.
40. Toulany M, Kasten-Pisula U, Brammer I, et al: Blockage of epidermal growth factor receptor-phosphatidylinositol 3-kinase-AKT signaling increases radiosensitivity of K-RAS mutated human tumor cells in vitro by affecting DNA repair. Clin Cancer Res 12: 4119-4126, 2006.

41. Toulany M, Kehlbach R, Florczak U, et al: Targeting of AKT1 enhances radiation toxicity of human tumor cells by inhibiting DNA-PKcs-dependent DNA double-strand break repair. Mol Cancer Ther 7: 1772-1781, 2008.

42. Mandic A, Viktorsson K, Heiden T, Hansson J and Shoshan MC: The MEK1 inhibitor PD98059 sensitizes C8161 melanoma cells to cisplatin-induced apoptosis. Melanoma Res 11: 11-19, 2001.

43. Smalley KS and Eisen TG: Farnesyl thiosalicylic acid inhibits the growth of melanoma cells through a combination of cytostatic and pro-apoptotic effects. Int J Cancer 98: 514-522, 2002 .

44. Spry M, Scott T, Pierce H and D'Orazio JA: DNA repair pathways and hereditary cancer susceptibility syndromes. Front Biosci 12: 4191-4207, 2007.

45. Cooke MS, Evans MD, Dizdaroglu M and Lunec J: Oxidative DNA damage: mechanisms, mutation, and disease. FASEB J 17: 1195-1214, 2003

46. Dhar A, Young MR and Colburn NH: The role of AP-1, NF-kappaB and ROS/NOS in skin carcinogenesis: the JB6 model is predictive. Mol Cell Biochem 234-235: 185-193, 2002.

47. Hunt CR, Sim JE, Sullivan SJ, et al: Genomic instability and catalase gene amplification induced by chronic exposure to oxidative stress. Cancer Res 58: 3986-3992, 1998.

48. Wong GH: Protective roles of cytokines against radiation: induction of mitochondrial MnSOD. Biochim Biophys Acta 1271: 205-209, 1995

49. Pommier Y, Barcelo JM, Rao VA, et al: Repair of topoisomerase I-mediated DNA damage. Prog Nucleic Acid Res Mol Biol 81: 179-229, 2006.

50. Sordet O, Redon CE, Guirouilh-Barbat J, et al: Ataxia telangiectasia mutated activation by transcription- and topoisomerase I-induced DNA double-strand breaks. EMBO Rep 10: 887-893, 2009. 